\title{
Real Time GPU-Based Fuzzy ART Skin Recognition
}

\author{
Mario Martínez-Zarzuela, Francisco Javier Díaz Pernas, \\ David González Ortega, José Fernando Díez Higuera, \\ and Míriam Antón Rodríguez
}

Higher School of Telecommunications Engineering

University of Valladolid (Spain)

mario.martinez@tel.uva.es

http://gti.tel.uva.es

\begin{abstract}
Graphics Processing Units (GPUs) have evolved into powerful programmable processors, becoming increasingly used in many research fields such as computer vision. For non-intrusive human body parts detection and tracking, skin filtering is a powerful tool. In this paper we propose the use of a GPU-designed implementation of a Fuzzy ART Neural Network for robust real-time skin recognition. Both learning and testing processes are done on the GPU using chrominance components in TSL color space. Within the GPU, classification of several pixels can be made simultaneously, allowing skin recognition at high frame rates. System performance depends both on video resolution and number of neural network committed categories. Our application can process 296 fps or 79 fps at video resolutions of $320 \times 240$ and $640 \times 480$ pixels respectively.
\end{abstract}

\section{Introduction}

Graphics Processing Units (GPUs) have been used for many years as CPU coprocessors, helping them in the task of rendering complex images onto the computer screen. Inside this special purpose processors, data downloaded from the $\mathrm{CPU}$ is transformed along the graphics pipeline. Inside this pipeline, several vertex and fragment processors can be programmed to perform user-defined calculations over the elements of a stream of data in a parallel fashion.

Human body parts detection has important applications as a first step in many high-level computer vision tasks such as personal identification, video indexing systems and human-machine interfaces (HMI). HMI needs a real-time video processing while consuming as few system resources as possible. Skin color segmentation on the GPU can be a first step towards robust and efficient HMIs.

James Fung et al. [1] was one of the first researchers who used GPUs in the field of computer vision. James Fung's open source OpenVidia project implements computer vision algorithms on computer graphics hardware. OpenVidia is able to make skin tone segmentation at $30 \mathrm{fps}$ at a resolution of $320 \mathrm{x} 240$ pixels, using RGB to HSV color conversion and threshold filtering.

In this paper, we propose a real time GPU-based Fuzzy ART Neural Network for skin recognition. The rest of this paper is organized as follows: for 
completeness, Section 2 summarizes Fuzzy ART architecture and training algorithm. Section 3 talks about the TSL color space and justify its convenience for skin color segmentation. Section 4 explains our GPU implementation of a Fuzzy ART skin recognizer. Section [5] summarizes the experimental results that were done for training the neural network and measuring the performance of the skin recognizer. Finally, Section 6 draws the main conclusions and outlines future research tasks.

\section{Fuzzy ART Neural Networks}

Fuzzy ART systems are able to categorize analog or binary input patterns, defined by $\mathrm{M}$ components. Each output neuron in the network represents a category and has an associated weight vector or long-term memory (LTM) trace $\boldsymbol{w}_{j}=\left(w_{j 1}, \cdots, w_{j M}\right)$. Initially, all weights are set to one, so each category is said to be uncommitted. When a category is first selected it becomes committed. For each input pattern $\boldsymbol{I}$ and output node $j$, the choice function, $T_{j}$, is defined by:

$$
T_{j}(\boldsymbol{I})=\frac{\left|\boldsymbol{I} \wedge \boldsymbol{w}_{\boldsymbol{j}}\right|}{\alpha+\left|\boldsymbol{w}_{\boldsymbol{j}}\right|}
$$

where the fuzzy MIN operator $\wedge$ is defined by $(\boldsymbol{p} \wedge \boldsymbol{q}) \equiv \min \left(p_{i}, q_{i}\right)$ and the norm $|\cdot|$ is defined by $|\boldsymbol{p}| \equiv \sum_{i=1}^{M}\left|p_{i}\right|$ for any $M$-dimensional vectors $\boldsymbol{p}$ and $\boldsymbol{q}$. The category choice is indexed by $J$, where $T_{J}=\max \left(T_{j}: j=1 \cdots N\right)$. Then, $\boldsymbol{w}_{\boldsymbol{J}}$ is said to be a fuzzy subset of $\boldsymbol{I}$ and it is fed down in order to measure its resemblance to the input pattern $\boldsymbol{I}$. The system enters in resonance if the match function meets the vigilance criterion $\rho$ :

$$
\frac{\left|\boldsymbol{I} \wedge \boldsymbol{w}_{\boldsymbol{J}}\right|}{|\boldsymbol{I}|} \geq \rho
$$

When this occurs and learning is enabled, vector $\boldsymbol{w}_{\boldsymbol{J}}$ is updated:

$$
\boldsymbol{w}_{J}^{\text {new }}=\beta\left(\boldsymbol{I} \wedge \boldsymbol{w}_{J}^{\text {old }}\right)+(1-\beta) \boldsymbol{w}_{J}^{\text {old }}
$$

Otherwise, node $J$ is inhibited and another node is selected. In case no node $j$ is found to meet the vigilance criterion, a new output neuron is committed. Proliferation of categories is avoided in Fuzzy ART by normalizing inputs. A useful rule for achieving normalization while preserving amplitude information is complement coding. If $\boldsymbol{a}$ represents the on-response of the pattern, each component of the off-response $\boldsymbol{a}^{c}$ is defined as $a_{i}^{c} \equiv 1-a_{i}$. Then, the complement coded input comes $\boldsymbol{I}=\left(\boldsymbol{a}, \boldsymbol{a}^{c}\right) \equiv\left(a_{1} \cdots a_{M}, a_{1}^{c} \cdots a_{M}^{c}\right)$ and $|\boldsymbol{I}|=M$ for every input pattern.

\section{Skin Color Segmentation TSL Space Color}

Color can be decomposed into one luminance and two chrominance components. Several researches have proved that skin colors have a certain invariance regarding 
chrominance components and in consequence skin tone and lighting mainly affect the luminance value 3 . Furthermore, normalized probability of skin colors can be modeled by a Gaussian distribution in the chromatic color space 3 .

Different color spaces separating chrominance and luminance components have been used for skin color segmentation (YIQ, YCbCr, CIE-Lab, CIE-Luv, HSV, IHS and TSL). In TSL color space 4, colors are specified in terms of Tint (T), Saturation (S) and Luminance (L) values. TSL has been selected as the best color space to extract skin color from complex backgrounds [5] because it has the advantage of extracting a given color robustly while minimizing illumination influence.

\section{GPU Implementation for Real Time Fuzzy ART Skin Recognition}

\subsection{Training}

An exhaustive description of a GPU-based Fuzzy ART Neural Network implementation for generic data learn and test can be found in [6]. For specific skin recognition application, complement coded TS features were chosen, so every input pattern is described as $\boldsymbol{I}=\left(\boldsymbol{a}, \boldsymbol{a}^{c}\right)=(T, S, 1-T, 1-S)$. By using complement coding we drastically reduce proliferation of categories and force $|\boldsymbol{I}|$ to be constant $(|\boldsymbol{I}|=M=2)$ for every input pattern, thus avoiding extra computing for calculating the match criteria (2). During learning process, each four-dimension input pattern $\boldsymbol{I}$ is replicated along a RGBA texture $I^{T}$. Four-dimension patterns can be stored in a single texel using its four different channels. Neural network weights are initialized to 1 and stored in another RGBA texture $W^{T}$.

Generalized size reduction operations on the GPU through a ping-pong technique [7, are used to obtain $\left|\boldsymbol{I} \wedge \boldsymbol{w}_{\boldsymbol{j}}\right|$ and Multiple Render Targets support allows to calculate the module of newer committed categories $\left|\boldsymbol{w}_{\boldsymbol{j}}\right|$ at the same time. The use of RGBA textures allows to run MIN and SUM operations on 4 -component vectors in one clock cycle on every fragment shader unit, making the process faster.

Reduced textures are then used to store the activity of each neuron, satisfying the match criteria, on the $\mathrm{R}$ channel of a texture $T^{T}$; $\mathrm{G}$ channel is used to store the category index; alpha channel takes the value of 1 if the match criteria is satisfied and 0 otherwise; finally, channel B can be used for printing the matching rate, which we found very useful for debugging purposes.

The $J_{\text {th }}$ neuron is found using a row reduction operation over texture $T^{T}$, where those fragments not satisfying the match criteria are discarded. If the system enters in resonance, the weights of the selected category are updated by rendering into the corresponding texel of the texture $W^{T}$ using scissoring 7 . If not, the new pattern is learned by rendering into a unused texel using equation (3).

\subsection{Testing}

For the real time skin recognition application, a GPU-based Fuzzy ART Neural Network implementation was developed. The system is able to read data from 
conventional USB Webcams using the DirectShow library. Webcam captures are directly downloaded to the GPU memory and stored in a texture, avoiding slow CPU-GPU data transfers. Each new frame is stored in the GPU as a bidimensional RGBA texture. Bidimensional textures are more amenable to be used on the GPU than one dimensional textures and this kind of organization maps specially well for skin pattern recognition in our computer vision application.

On each frame, several computations take place in order to recognize skin tone pixels. Before the neural network can start recognition process, a pre-processing stage has to be configured in order to translate 3-color dimension RGB coded frames into 4 color dimension complement coded ones. A shader in this stage uses R,G and B components to calculate $\mathrm{T}$ and $\mathrm{S}$ chrominance components and its corresponding complements $1-\mathrm{T}$ and 1-S, and renders them to a RGBA texture $T S^{T}$. Each texel on the new texture has the whole information we need to categorize each pixel of the original image. Weights learned during training process are loaded into a unidimensional $W^{T}$ RGBA texture. Each texel on this texture stores a long-term-memory trace $\boldsymbol{w}_{\boldsymbol{j}}$.

During neural network categorization, several input patterns stored in texture $T S^{T}$ can be categorized in a parallel fashion using every fragment processor available on the GPU. Category choice occurs through the execution of a shader for $N$ times, being $N$ the number of categories in the neural network. In each pass, the activation of the $j_{\text {th }}$ output neuron and the match function are computed for every input pattern. This values are rendered into a RGBA output texture, which is used as input for the next iteration, using a ping-pong technique. The other two channels in the output texture are used for indexing the selected category of the pattern and counting the number of categories the input pattern has been tested against.

If the activation in pass $j$ is bigger than the computed activation in pass $j-1$ and the match criterion is satisfied, then the index category is updated on the output texture. Rendering both the index of the selected category and the match function to the output texture allows the expert to visually analyze results. In our implementation, different gray levels on channel $\mathrm{R}$ belong to different skin categories committed during training process, alpha channel represents the level of resemblance of that pixel in the original image to the selected skin category.

For real-time demonstration of the system, another post-processing stage is used. In this last stage a shader is configured to render onto the screen just those pixels in the original image which have crossed all the tests and have been recognized as skin. Final result is an image were only skin recognized regions are drawn over a uniform color background.

\section{$5 \quad$ Experimental Results}

\subsection{Statistical Skin Color Selection}

From two different image databases, regions containing skin pixels were selected. Normalized frequency of TSL chrominance components of every pixel in these regions was graphically represented. 
Afterwards, we estimated the skin color distributions as a normal distribution through the minimum covariance determinants estimator (MCD) [8]. A color was classified to belong to skin if its mahalanobis distance to the mean color given by the mean vector of the modeled distribution is lower than a threshold defined through the critical value of $\alpha \%$ of a $\chi^{2}$ distribution with two degrees of freedom. From previous works [9], we concluded that the best results are achieved with the threshold value of 0.99 .

We created 2 different Training Sets of colors for the neural network. Training Set 1 is formed by all the colors selected as skin colors from the skin regions in the 3056-image Faces96 database [10, a well-known face database and Training Set 2 from the skin regions in a 4176-image private database (taken by us with different cameras and lighting conditions and including not only faces but hands and arms).

\subsection{Fuzzy ART Off-Line Training}

From the colors selected in the two training sets, the input features to train the Neural Network were the T and S components and their complementary values. Performance of our GPU-based Fuzzy ART implementation relays on several factors: length of the input pattern $\boldsymbol{I}$, number of input patterns $P$ presented to the network and number of created categories $N$. During the learning process, the number of committed categories varies depending both on the grade of similarity between patterns and the vigilance criterion $\rho$. The number of input features used during training were 671438 for Training Set 1 and 559640 for Training Set 2. The network was trained using $\alpha=0.001$ and different values for $\rho$ in the range $[0.90 ; 0.97]$. Table 1 subsumes the number of committed categories in each Training Set with different $\rho$ values. The bigger the value of $\rho$ is, the bigger the number of categories created by the network is. Although the number of colors is very big, few categories are created proving both training colors and selection of TS features were correct.

Table 1. Number of committed categories with different $\rho$

\begin{tabular}{c|r|r|r|r}
$\rho$ & $\mathbf{0 , 9 0}$ & $\mathbf{0 , 9 3}$ & $\mathbf{0 , 9 5}$ & $\mathbf{0 , 9 7}$ \\
\hline Training Set 1 & 9 & 15 & 33 & 71 \\
Training Set 2 & 8 & 17 & 24 & 73
\end{tabular}

\subsection{Fuzzy ART Real-Time Testing}

In order to measure the performance of our implementation, several tests were done on a dual-core $3.2 \mathrm{GHz}$ Pentium 4 with 1GB RAM, GeForce 7800GTX $256 \mathrm{MB}$ GPU (containing 24 fragment processors) and a generic webcam able to capture at resolutions of $640 \times 480$ pixels at a rate up to $90 \mathrm{fps}$.

Figure 1 shows different skin recognition results for the same image using the weights learned from Training Set 1. Figure 1(a) shows the original captured RGB image. Figure 1(f) represents the input features introduced to the network. 


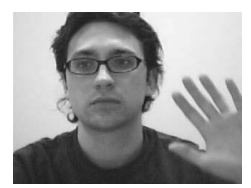

(a) RGB

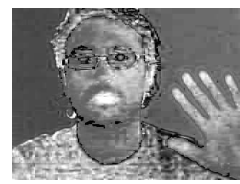

(f) TS

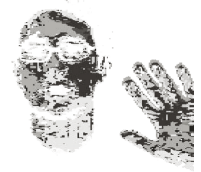

(b) $\rho=0.90$

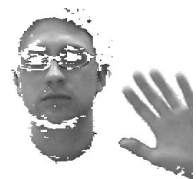

(g) $\rho=0.90$

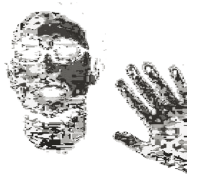

(c) $\rho=0.93$

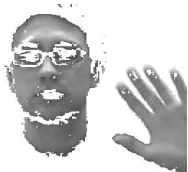

(h) $\rho=0.93$

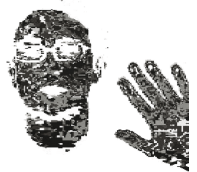

(d) $\rho=0.95$

(e) $\rho=0.97$

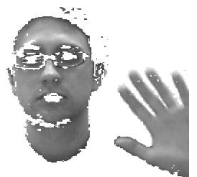

(i) $\rho=0.95$
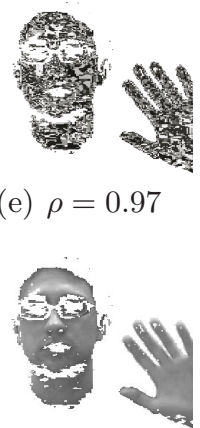

(j) $\rho=0.97$

Fig. 1. Selected categories and segmented skin regions for a single image using Training Set 1 and different $\rho$ values

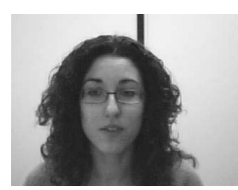

(a) RGB

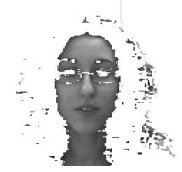

(b) Tr. $\rho=0.90$

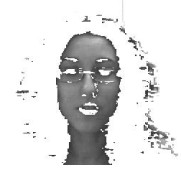

(c) Tr. Set-1 $\rho=$ (d) Tr.

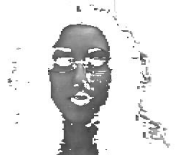

$\rho=0.95$

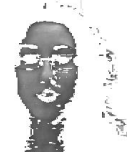

Set-1 (e) Tr. Set-1 $\rho=$ 0.97

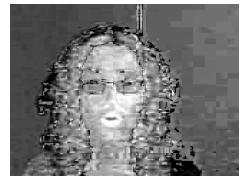

(f) $\mathrm{TS}$

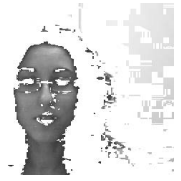

(g) $\operatorname{Tr}$. $\rho=0.90$

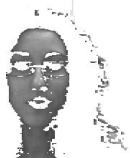

Set-2 (h) Tr. $\rho=0.93$
Set-2 (i) 0.95
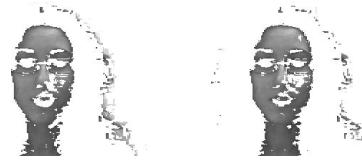

Fig. 2. Testing results with Training Sets 1 and 2

The image was created by writing on every RGB channel the tone $(\mathrm{T})$ value in each pixel and choosing A channel to be the value of the saturation characteristic (S). Figures from 1(b)to $1(\mathrm{e})$ show regions identified as different skin categories by the network with $\rho$ increasing from 0.90 to 0.97 respectively. Finally, figures from $1(\mathrm{~g})$ to $1(\mathrm{j})$ show the final filtered image in which only skin recognized pixels are drawn on a white background.

Figure 2 depicts the different behavior of the network with the two different Training Sets. For low $\rho$ values false positive rate is bigger with Training Set 2, making the recognition unacceptable for $\rho=0.9$. For increasing values of $\rho$ false positive rate decreases on both Training Sets, while hit rate significantly worsen only in Training Set 2.

Table 2 shows frame rate accomplished by the neural network on the GPUs for different video resolutions. As value of $\rho$ and resolution increase, frame rate 
Table 2. Frames per second for different resolutions and $\rho$ values

\begin{tabular}{|c|c|c|c|c|c|c|}
\hline & & & & & 040 & \\
\hline & $0.900,95$ & 0,97 & $0.900,95$ & 0,97 & $0.900,95$ & 0,97 \\
\hline Color & 270 & 42 & 212 & 32 & 71 & 11 \\
\hline Color set 2 & $296 \quad 120$ & 43 & 230 & 31 & 79 & 10 \\
\hline
\end{tabular}
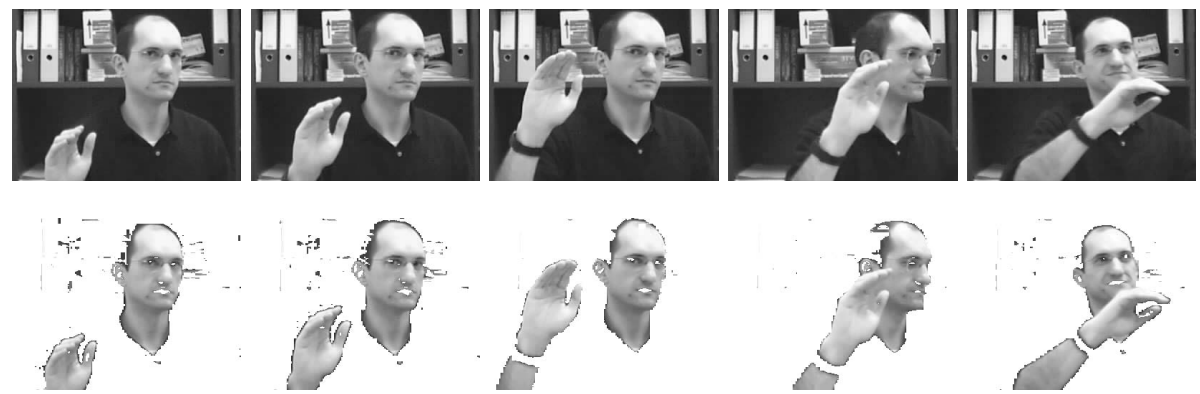

Fig. 3. Skin recognition on a video sequence at $270 \mathrm{fps}$ with Training Set 1

decreases. Best performance, which was achieved using a resolution of $320 \times 240$ pixels and $\rho=0.90$, is $296 \mathrm{fps}$. The number of frames that can be processed by the network strongly depends on the number of committed categories every pixel has to be tested to.

Figure 3 shows the performance of the neural network with 5 frames extracted from a video sequence, using Training Set 1 and $\rho=0.90$. With this value of $\rho$ some background pixel are wrongly categorized but the hit rate is very high, achieving an excellent real-time skin recognition.

\section{Conclusions}

An implementation of a GPU-based Fuzzy ART Neural Network for real time skin recognition was introduced in this paper. Our design successfully faces the problem of using a neural network for pattern classification when time is a major requirement. A robust and complete set of skin colors and a good selection of input features (chrominance components of TSL color space) is necessary to train the network so that it can recognize skin in real changing conditions.

Experimental results show our system achieves excellent skin tone pixels recognition at high frame rates with an $n$ VIDIA 7800GTX GPU equipped video card, which includes 24 fragment shaders in the pipeline. GPUs are quickly evolving and every few months a new generation of improved processors is made publicly available. Forward compatibility of our design is guaranteed and we can expect an incredible performance with newer cards, such as the recently appeared NVIDIA GeForce 8800, which incorporates 128 unified shaders.

Fuzzy ART skin recognition using a fast GPU can be the first high-computational-cost but low-time-consuming step in a much more complex system for 
computer vision. In this sense, our design is potentially applicable in a wide range of applications, such as human-machine interfaces or video indexing.

\section{References}

1. Fung, J.: Computer vision on the gpu. In: Pharr, M. (ed.) GPU Gems 2, pp. 649665. Addison-Wesley, Reading (2005)

2. Carpenter, G.A., Grossberg, S., Rosen, D.B.: Fuzzy ART: Fast stable learning and categorization of analog patterns by an adaptive resonance system. Neural Networks 4(6), 759-771 (1991)

3. Hsieh, I.S., Fan, K.C., Lin, C.: A statistic approach to the detection of human faces in color nature scene. Pattern Recognition 35(7), 1583-1596 (2002)

4. Terrillon, J., David, M., Akamatsu, S.: Automatic detection of human faces in natural scene images by use of a skin color model and of invariant moments. In: Proceedings of the IEEE Conference on Automatic Face and Gesture Recognition, pp. 112-117. IEEE Computer Society, Washington, DC, USA (1998)

5. Duan-sheng, C., Zheng-kai, L.: A novel approach to detect and correct highlighted face region in color image. In: AVSS '03: Proceedings of the IEEE Conference on Advanced Video and Signal Based Surveillance, pp. 7-12. IEEE Computer Society, Washington, DC, USA (2003)

6. Martínez-Zarzuela, M., Díaz Pernas, F., Díez Higuera, J., Antón Rodríguez, M.: Fuzzy ART neural network parallel computing on the gpu. In: Sandoval, F. (ed.) IWANN 2007. LNCS, vol. 4507, pp. 463-470. Springer, Heidelberg (2007)

7. Pharr, M. (ed.): GPU Gems 2 (Programming Techniques for High-Performance Graphics and General-Purpose Computation). Addison-Wesley, Reading (2005)

8. Rousseeuw, P.J., Driessen, K.V.: A fast algorithm for the minimum covariance determinant estimator. Technometrics 41(3), 212-223 (1999)

9. González Ortega, D., Díaz-Pernas, F., Díez-Higuera, J., Martínez-Zarzuela, M., Boto Giralda, D.: Computer vision architecture for real-time face and hand detection and tracking. In: Bres, S., Laurini, R. (eds.) VISUAL 2005. LNCS, vol. 3736, pp. 35-49. Springer, Heidelberg (2006)

10. Spacek, L.: Faces96 database:

http://cswww.essex.ac.uk/mv/allfaces/faces96.html 